\title{
Magnetic Resonance Studies of the Origin of Ferromagnetism in $\mathbf{G a}_{1-x} \mathbf{M n} \mathbf{n}_{x} \mathbf{A s}$
}

\author{
O.M. Fedorych ${ }^{a}$, Z. Wilamowski ${ }^{a}$, M. Potemski ${ }^{b}$, \\ M. BYsZEWSKI ${ }^{b}$ AND J. SADOWSKI ${ }^{c, a}$ \\ ${ }^{a}$ Institute of Physics, Polish Academy of Sciences \\ al. Lotników 32/46, 02-668 Warsaw, Poland \\ ${ }^{b}$ Grenoble High Magnetic Field Laboratory, MPI/CNRS \\ 380-42 Grenoble, France
}

${ }^{c}$ Niels Bohr Institute, Copenhagen University, 2100 Copenhagen, Denmark

Different types of magnetic resonance observed in $\mathrm{Ga}_{1-x} \mathrm{Mn}_{x}$ As reflect three different magnetic phases: para-, ferro-, and ferrimagnetic. Ferromagnet is characterized by single isotropic resonance line. A complex spectrum in ferrimagnet can be described by $g$ factor equal to 1.44 and a sum of an axial and cubic anisotropy field. The axial field is by an order of magnitude greater than the cubic one. The complex structure of ferrimagnetic resonance is attributed to spin-wave resonance. Quantitative analysis of the dispersion of spin wave shows that the range of exchange coupling is very long, of the order of $25 \mathrm{~nm}$, while spin-wave stiffness and the total exchange field are very small. The exchange field as evaluated from spin wave is by two orders of magnitude smaller than the Zener field corresponding to the critical temperature.

PACS numbers: 71.55.Eq, 76.30.Fc, 75.50.Pp, 75.30.Gw

\section{Introduction}

GaMnAs is a classical semiconductor, where Mn impurities play the role of acceptors. The $\mathrm{Mn}^{2+}$ ions have well localized $S=5 / 2$ spins and the ionized holes are characterized by the magnetic moment $j=3 / 2$. In diluted magnetic $\mathrm{Ga}_{1-x} \mathrm{Mn}_{x}$ As free carriers play the basic role in occurrence of the ferromagnetism: they mediate the exchange between localized Mn spins [1-3]. The occurrence of an ordered magnetic phase of $\mathrm{Ga}_{1-x} \mathrm{Mn}_{x}$ As has been well proven. The critical temperature exceeds $110 \mathrm{~K}$. 


\section{Results}

Magnetic resonance technique allows to clarify some details of spin interactions and to distinguish various magnetic structures. Para-, ferro-, and ferrimagnetic spectra of $\mathrm{Ga}_{1-x} \mathrm{Mn}_{x}$ As were obtained for different $\mathrm{Mn}$ concentrations. Figure 1 shows critical temperatures obtained from the analysis of temperature dependence of resonance amplitude as a function of $x$, different symbols corresponding to different magnetic phases. For low Mn concentration, $x<0.01$, the paramagnetic resonance is observed. For $x<0.002$ well resolved fine and hyperfine structures prove that there is no correlation of precession of individual Mn spins [4]. An increase in Mn concentration leads to exchange narrowing of structures and a single isotropic resonance line is observed.

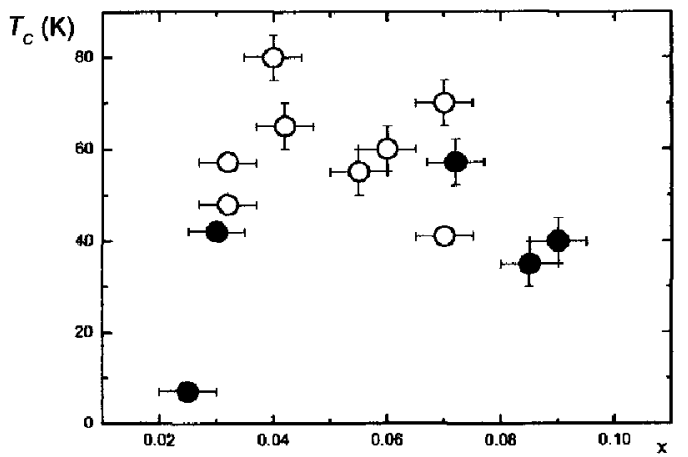

Fig. 1. The dependence of the critical temperature, evaluated from magnetic resonance studies, on Mn concentration, $x$. Full and open circles correspond to ferromagnetic and ferrimagnetic samples, respectively.

At larger Mn concentration $\mathrm{Ga}_{1-x} \mathrm{Mn}_{x} \mathrm{As}$ exhibits ferromagnetic properties. There is no evident change of the character of resonance: a single isotropic line with $g$ factor very close to 2 is observed. The only difference is the behavior of the temperature dependence of the resonance amplitude. The ferromagnetic phase observed in the $\mathrm{Mn}$ concentration range $0.01<x<0.03$ coincides with the occurrence of the phase of the Mott insulator. The shift of ferromagnetic resonance is due to the sample demagnetization and is weak [5] because of small sample magnetization for diluted systems. On the other hand, the mean $p-d$ exchange field, so-called Zener field [2] or Knight shift, caused by spin polarized holes is expected to be very large, of the order of many tesla. The fact that this shift is not observed can be related to strong disorder in insulating samples. The resulting spin relaxation of hole spins is very fast, much faster than the Larmor frequency, and the direction of the hole polarization follows the direction of magnetic moment 
of Mn spins, $\boldsymbol{M}$. In such a case, $p-d$ field being parallel to $\boldsymbol{M}$ does not affect its precession frequency $[6,7]$.

The insulator to metal transition occurring at $x \approx 0.03$ is followed by a change of the magnetic structure. For metallic phase occurring in the range $0.03<x<0.07$ a ferrimagnetic resonance is observed. Moreover, reentrance to insulating phase at $x \approx 0.07$ is accompanied by the reentrance to ferromagnetic phase. This coincidence shows the dependence of magnetic structure on electrical phase.

An example of the angular dependence of ferrimagnetic resonance spectra is shown in Fig. 2. The structure in Fig. 2b originates from spin-wave (SW) resonances. The high field edge of SW structure corresponds to the uniform mode of the ferrimagnetic resonance [8,9]. The dependence of the resonance field $H_{0}$ of the direction of applied magnetic field, $\theta$, is plotted as squares in Fig. $2 . H_{0}$ for

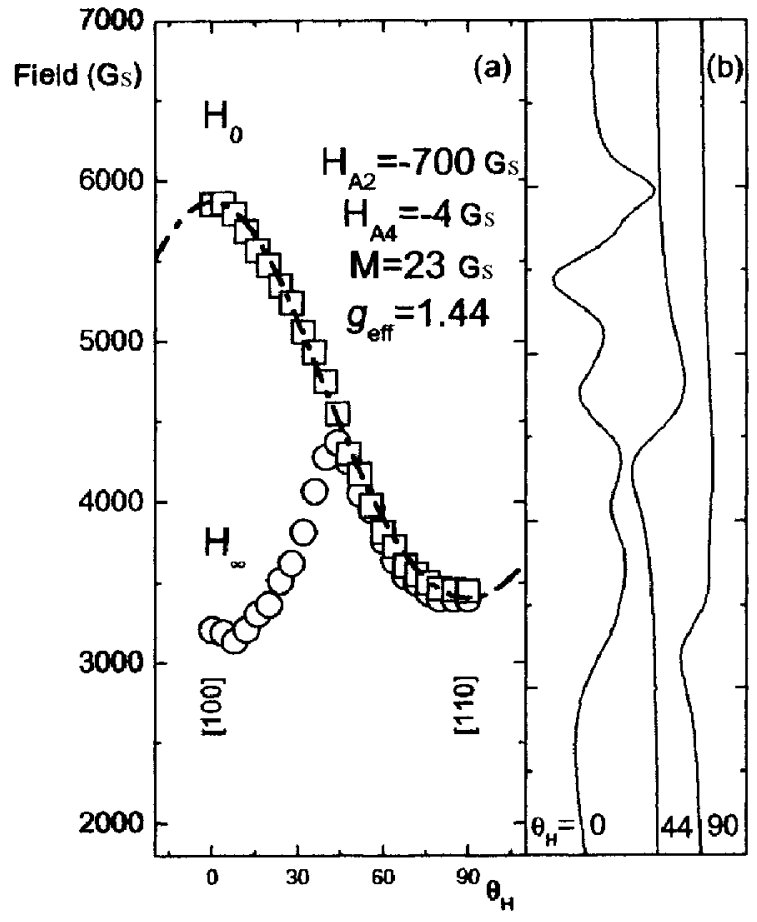

Fig. 2. (a) The dependence of resonance position as a function of angle between the direction of applied magnetic field and normal to the film plane, $\theta$. Squares correspond to uniform mode of magnetic resonance, $H_{0}$. Circles stand for the low field limit of SW structure, $H_{\infty}$, which is seen as a broad line. Dashed-dot line presents the fitted angular dependence. (b) Resonance spectra measured for different orientations of applied magnetic field. 
the perpendicular orientation, $\theta=0$, is higher by $3 \mathrm{kGs}$ than for the in-plane orientation of magnetic field, $\theta=90^{\circ}$. This indicates that the in-plane orientation is the easy plane of magnetization.

\section{Discussion}

The anisotropy presented in Fig. 2b can be described using classical analysis within the formalism of anisotropy fields $[10,11]$. The resonance frequency, $\omega$, is given by second derivatives of magnetic free energy $U$ over the spherical coordinates of the magnetization vector

$$
\frac{\omega}{\gamma}=\frac{1}{M \sin \theta}\left[\frac{\partial^{2} U}{\partial \theta^{2}} \frac{\partial^{2} U}{\partial \varphi^{2}}-\left(\frac{\partial U}{\partial \theta \partial \varphi}\right)^{2}\right]^{1 / 2}
$$

Here $\gamma$ - gyromagnetic ratio. Values of azimuthal, $\varphi$, and polar $\theta$ angles should satisfy the condition of full energy minima $\partial U / \partial \theta=\partial U / \partial \varphi=0$. The energy of spin system, $U$, can be approximated by the expression

$$
\begin{aligned}
U= & -M H\left[\cos \theta \cos \theta_{H}+\sin \theta \sin \theta_{H} \cos \left(\varphi-\varphi_{H}\right)\right] \\
& -4 \pi M \cos \theta+K_{2} \sin ^{2} \theta+\frac{1}{4} K_{4}\left(\sin ^{2} 2 \theta+\sin ^{4} \theta \sin ^{2} 2 \varphi\right),
\end{aligned}
$$

where $H$ stands for the magnitude, while $\varphi_{H}$ and $\theta_{H}$ for the azimuthal and the orbital angle of applied magnetic field. First term corresponds to the Zeeman energy, second reflects the shape anisotropy caused by magnetic dipole-dipole interaction, third describes the axial anisotropy caused by the layer strain, and the last term corresponds to the cubic anisotropy. $K_{2}$ and $K_{4}$ are axial and cubic anisotropy energies.

The dash-dot line in Fig. 2a has been plotted for the fitting values of the axial and the cubic anisotropy fields $H_{\mathrm{A} 2}=K_{2} / M=(-700 \pm 10)$ Gs and $H_{\mathrm{A} 4}=$ $K_{4} / M=(-4 \pm 2) \mathrm{Gs}$, respectively. For $x=0.07$ the demagnetization field has been estimated to be $4 \pi M \approx 290$ Gs. Both anisotropy fields vary from sample to sample. Generally, however, the axial anisotropy dominates over the cubic one and varies in the range $300 \mathrm{Gs}$ to $1 \mathrm{kGs}$.

The classical analysis of the anisotropy presented above is simplified and does not reflect all details and peculiarities of the magnetic anisotropy of $\mathrm{Ga}_{1-x} \mathrm{Mn}_{x} \mathrm{As}$. Detailed measurements of the magnetic remanent magnetization [12] and of the magnetic resonance [13] show that an anisotropy term corresponding to the anisotropy of a symmetry lower than tetragonal should be additionally considered.

The evaluated value of $g=1.44$ well confirms that the precessing moment is a vector sum of Mn spins, characterized by $g=2$, and of the hole spin with a smaller $g$-factor. Our observation of the low frequency mode of the ferrimagnetic resonance proves that both subsystems are ordered and strongly coupled to each other [9]. 


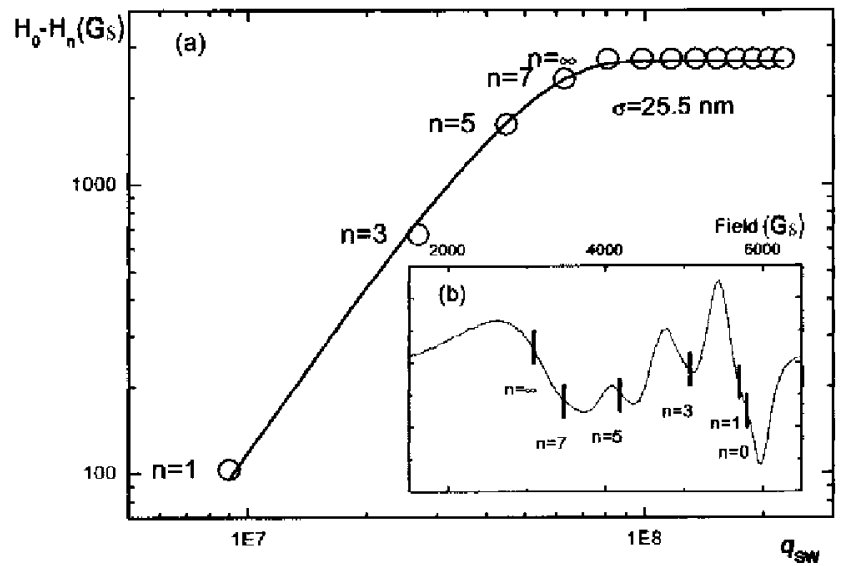

Fig. 3. (a) Dispersion dependence of spin waves. (b) $9.44 \mathrm{GHz}$ resonance spectra obtained at $T=2 \mathrm{~K}$ in normal configuration. A complex wave structure corresponds to the SW resonance. Numbers indicate the positions of the consecutive spin waves.

The ferrimagnetic resonance is accompanied by well resolved spin-wave resonances. The SW structure is shown in Figs. 2b and 3b. Similar SW structures have already been observed [13-17]. Previous reports underscore a peculiarity of the observed SW structure. In this paper we present a new concept of the interpretation of the SW structure. We assume that the exchange interaction is described by a Gaussian function of the exchange range. The dependence can be parametrized by two phenomenological quantities: the range of interaction, $\sigma$, and the force of the exchange coupling $J_{0}$. The theoretically calculated SW dispersion is shown in Fig. 3 by the solid curve. An excellent fit of SW dispersion was obtained. The shift of the SW resonance field, $H_{n}-H_{0}$, directly related to the energy of SW, $E_{\mathrm{SW}}=g \mu_{\mathrm{B}}\left(H_{0}-H_{n}\right)$, is plotted as $q$-vector of SW, $q_{\mathrm{SW}}$. The index $n$ corresponds to the number of half wave length of the standing wave resonating between the layer walls. The broad line which is observed at low magnetic field corresponds to the saturation of the SW dispersion and is equal to the double value of the total exchange field acting on a mean Mn spin, $H_{0}-H_{\infty}=2 J_{0} z / g \mu_{\mathrm{B}}$, where $z$ is the number of Mn spins within the sphere of the radius $\sigma$. The length of exchange interaction can be directly evaluated from the $q_{\mathrm{SW}}$ vector corresponding to the occurrence of SW energy saturation, $q_{\mathrm{SW}} \cong 1 / \sigma$.

\section{Conclusions}

The values of the obtained parameters are rather surprising. The total exchange field acting from $z=0.26 \times 10^{6} \mathrm{Mn}$ spins is $z J_{0} / k_{B}=0.29 \mathrm{~K}$. It is by two orders of magnitude smaller as compared to the critical temperature, $T_{\mathrm{C}}=35 \mathrm{~K}$. 
This comparison shows that the Zener field but not the Heisenberg Mn-Mn interaction is the dominant origin of magnetic order in $\mathrm{Ga}_{1-x} \mathrm{Mn}_{x} \mathrm{As}$. Zener field induces the magnetic order, but does not affect the SW propagation, which is ruled by the exchange coupling with neighboring spins only. Also the evaluated range of the exchange interaction is very long, $\sigma \approx 25 \mathrm{~nm}$. We relate it to a small concentration of the spin minority holes.

\section{Acknowledgments}

This work was supported by the State Committee for Scientific Research (Poland) under grant PBZ-KBN-044/P03/2001 and grant 2 P03B 05423.

\section{References}

[1] H. Ohno, J. Magn. Magn. Mater. 200, 110 (1999).

[2] T. Dietl, H. Ohno, F. Matsukura, J. Cibert, D. Ferrand, Science 287, 1019 (2000).

[3] For a review see T. Dietl, Semicond. Sci. Technol. 17, 377 (2002).

[4] O. Fedorych, E. Hankiewicz, Z. Wilamowski, J. Sadowski, Phys. Rev. B 66, 045201 (2002).

[5] J. Szczytko, A. Twardowski, K. Świątek, M. Palczewska, M. Tanaka, T. Hayashi, K. Ando, Phys. Rev. B 60, 8304 (1999).

[6] H. Hasegawa, Prog. Theor. Phys. 21, 483 (1959).

[7] S.E. Barnes, Adv. Phys. 30, 801 (1981).

[8] M. Farle, Rep. Prog. Phys. 61, 755 (1998).

[9] S. Foner, in: Magnetism, Eds. G.T. Rado, H. Shul, Academic Press, New York 1963, p. 381.

[10] A.G. Gurevich, in: Magnetic Oscillations and Waves, Science, Moskva 1994, p. 464.

[11] C. Kittel, Phys. Rev. 110, 4 (1958).

[12] M. Sawicki, F. Matsukura, A. Idziaszek, T. Dietl, G. Shott, C. Ruester, G. Karczewski, G. Schmidt, L. Molenkamp, cond-mat/0212511, submitted to Phys. Rev. $B$.

[13] X. Liu, Y. Sasaki, J.K. Furdyna, Phys. Rev. B 67, 205204 (2003).

[14] S.T.B. Goennenwein, T. Graf, T. Waaner, M.S. Brandt, M. Stutzmann, Appl. Phys. Lett. 82, 5 (2003).

[15] Mark Rubinstein, A. Hanbicki, P. Lubitz, M. Osofsky, J.J. Krebs, B. Jonker, J. Magn. Magn. Mater. 250, 164 (2002).

[16] O. Fedorych, M. Byszewski, Z. Wilamowski, M. Potemski, J. Sadowski, Acta Phys. Pol. A 102, 617 (2002).

[17] O. Fedorych, M. Byszewski, Z. Wilamowski, M. Potemski, J. Sadowski, J. Supercond. 16, 1 (2003). 